\title{
إستقلال غينيا
}

عام 1901

إعداد الباحثة

نادية عيد السمان عبد الجواد 



\section{استقلال غينيا عام 1901}

\section{ملخص:}

يهدف هذا البحث إلي دراسة أهمية استقلال غينيا والمراحل التي مهدت إلى الحصول على هذا الاستقلال وأثثر على العلاقات بين غينيا وفرنسا ـ وقد نتاولت الدراسة الموقف في فرنسا منذ مجئ ديجول إلي الحكم في مايو 1901 ، وجولته بين المستعمرات الأفريقية في الفترة ما بين اب-דr أغسطس 1901 لعرض الاستور الفرنسي الجديد لتحديد العلاقة بين فرنسا ومستعمراتها في القارة الأفريقية . وقد أوضحت الدراسة الانقسام بين الأحزاب الأفريقية من خلال عرض موققها من

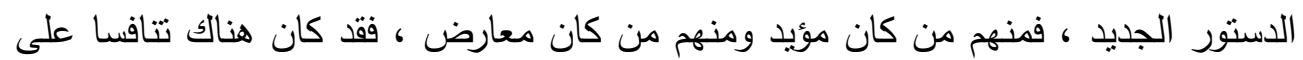
الزعامة الأفريقية في أفريقيا الغربية الفرنسية ، وكانت غينيا تدعو إلي الوحدة بين الأحزاب وتحديد موقفهم من الدستور وذلك برفضه في الاستفتاء المقترح في Y سبتمبر عام 1901 1، ولكنها خرجت في النهاية وحيدة وقام الثعب الغيني برفض الدستور على الرغم من

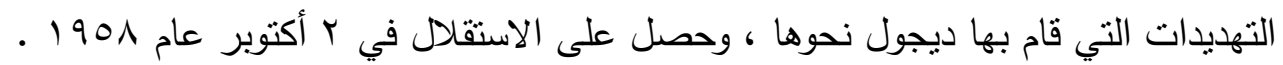
وقد أظهرت نتائج الدراسة أهمية التكتل والوحدة بين الأفريقيين التي دعا اليها سيكوتوري قبل الاستقلال ولكنه فنشل في ذلك ، وتحقق هدفه بعد الاستقلال من خلال الاتحاد بين غانا وغينيا في نوفمبر عام 1901 ـ الئرئ 


\section{Independence of Guinea in 190^}

\section{Abstract:}

This research aims to study the importance of the Independence of Guinea and its impact on the relations between Guinea and France . This study has taken the situation in France Since de Gaulle's coming to power in May 1901, and his trip between African colonies during the period between August $r$ to $r q, 1901$ to view the new French constitution, and to determine the relationship between France and her colonies in the African continent .

The study showed the split between African parties through the presentation of its position on the new constitution, some of whom were supporters and some of them were shows, there has been competition for African leadership in French West Africa, Guinea was calling for unity between the parties and determine their position on the Constitution by refusing it in the referendum in the September $\wedge^{\wedge}$, 190^, but it came out single in the end and the Guinean people has rejected the Constitution in spite of threats made by de Gaulle so, and got independence on October $r$, $190 \wedge$.

The results showed the importance of unity among Africans, which Sékou Touré called for it before independence, but he failed to do so, and achieved his goal after independence through the union between Ghana and Guinea in November $190 \wedge$. 
لقد عانت الجمهورية الفرنسية الرابعة انقسامات سياسية داخلية أنعكس أثزها على السياسة الخارجية لفرنسا ، وتميزت تلك الفترة بعدم الاستقرار ، حيث أدت الأزمات الوزارية إلى تغيير الحكومة عشرين مرة . (') وفي با مايو عام 1901 قامت بمحاولة الانقلاب العسكري في الجزائر ، واستولى اليمنيون في الجيش الفرنسي على الجزائر ، وسقطت أخر حكومة في الجمهورية الرابعة

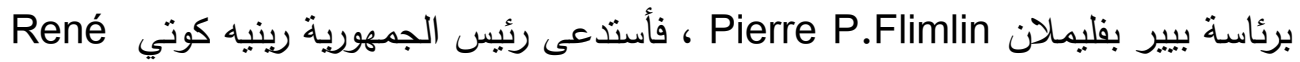
Charles De Gaulle الجنرال شارل ديجول Coty الذي كان قد أعتزل الخدمة منذ مدة طويلة - لكي يتولى رئاسة الحكومة ، وكان على استعداد لتلبية النداء ـ (؟نائل وبالفعل تقدم ديجول إلى الجمعية الوطنية وطلب ثقتها على برنامج حكومته ، فطلب موافقتها على منحه سلطات كاملة لمدة ستة أثنهر لإعادة النظام والوحدة بين الفرنسيين(")، منحه سلطة تعديل الدستور ، وصرح بأن تعديل الدستور سيشمل علاقات فرنسا بالثعوب

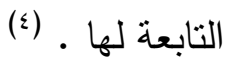
وبعد أن ناقتت الجمعية الوطنية البرنامج منحته التقة بأغلبية وبr صوتا ضد

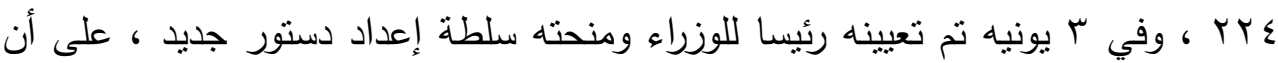

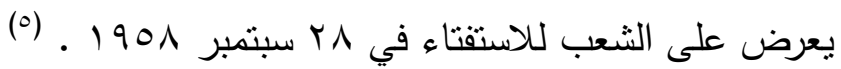
الاستور الفرنسي الجديد عام

وقد نصت بنود هذا الدستور على أن تُكون فرنسا مع الجمهورية الأفريقية التي تقبل هذا الدستور رابطة الجماعة الفرنسية أو الاتحاد الفرنسي(French Community ) وهو ال

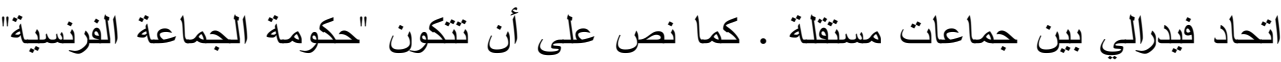
من رئيس الجمهورية الفرنسية ومندوب عن كل جمهورية من جمهوريات الجماعة ، وسكرتير

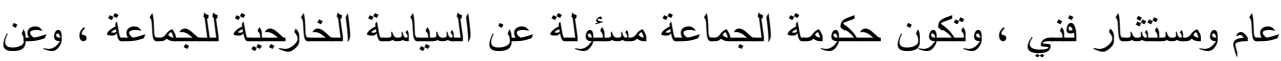
شُئون الدفاع ، وإصدار العملة ، و الثئون الاقتصادية العامة والتعليم العالي ، وفيما عدا

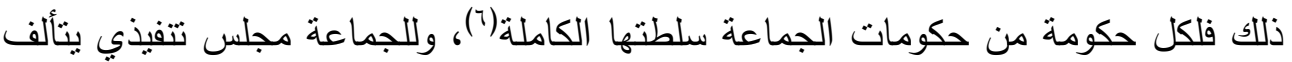


من رؤساء حكومات الجماعة(") ، ويكون للجماعة مجلس شيوخ يتألف من مندوبين عن

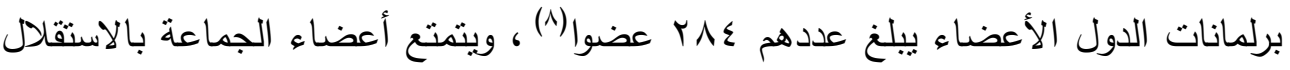

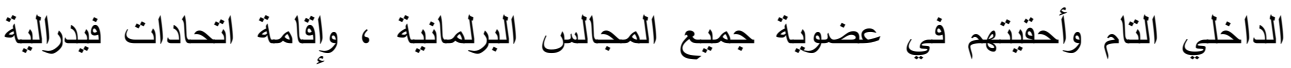

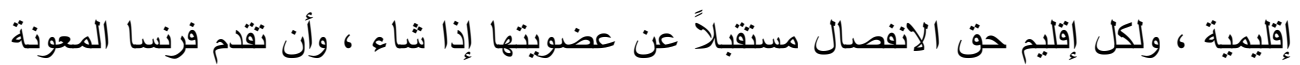

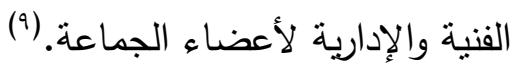
موقف الأحزلب الأفريقية من الاستور الفرنسي الجديد :

Rassemblement Démocratique حزب الاتحساد الديمقراطى الأفريقى

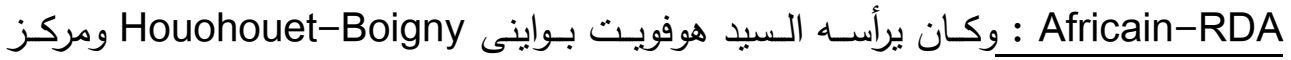

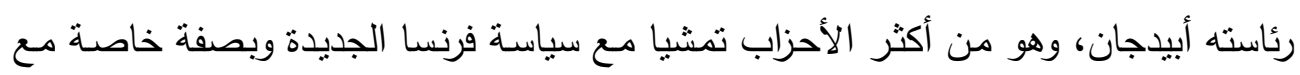

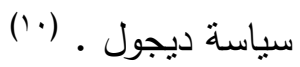
وينقسم الحزب إلى اتجاهين ، الاتجاه الأول : وهو الجناح الأيمن للحزب ويطالب التاب

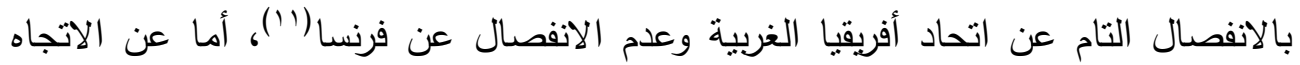

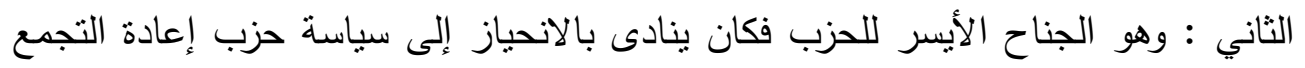

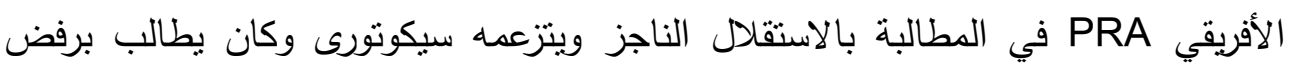
الاستور مالم ينم تعديله قبل الاستفتاء لينص صراحة على التنظيم الفيدرالي في أفريقيا ويتم على أساسه الاستقلال والارتباط مع فرنسا ـ (ir) حزب إعادة النجمع الأفريقى Parti Regroupement Africani-PRA : ويرأسه ليوبولد سنغور Leopold Senghor وكان يطالب بقبول الدستور ثم إتمام إجراءات الوحدة الفيدرالية قبل إعلان الاستقلال .r(r)

وقد صرح سنغور في مؤتمر الحزب المنعقد في كوتونو Cotonou بمقاطعة داهومي في Y يوليو 1901 بهذه المطالب ، وقد حضر هذاب المؤتمر ثلاثمائة مبعوث

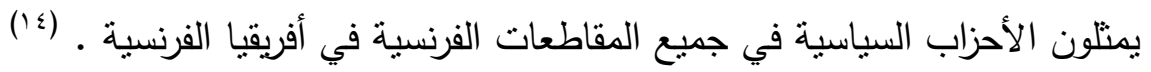

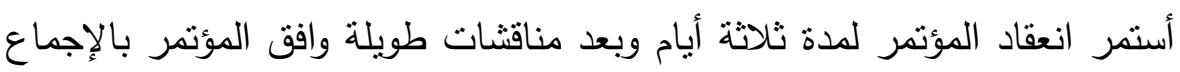

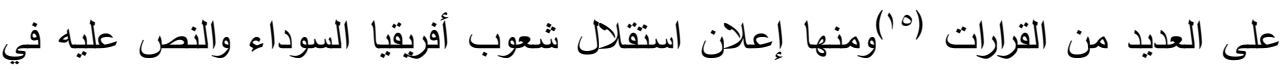


الدستور الفرنسي الجديد؛ إقامة وحدة أفريقيا السوداء وإزالة الحدود غير الطبيعية ؛ تكوين جمعية تشريعية تكون مهمتها تحديد حدود الدولة الجديدة ونوع الحكم والنظام الذي يصلح

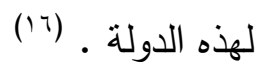

وفي النهاية انتهى بيان المؤتمر بالاتي : " يصادق المؤتمر على شعار الاستقلال

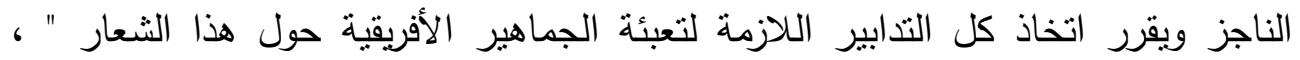
وهكذا أُعلن من كوتونو حرب شعوب أفريقيا السوداء على حكومة الجنرال ديجول الفرنسية. ( الفكان)

: Mouvement Républician Populaire - MSA الحركة الاشتراكية الأفريقية

اجتمت الأحزاب الاشتراكية الأفريقية في شهر أغسطس بكوناكري عاصمة غينيا واتفقت فيما بينها على توحيد برنامجها السياسي ، وطالبت فرنسا بتغيير وضعها ومنح الأقاليم

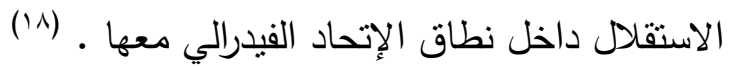

\section{رحلة ديجول في المستعمرات الافريقية :}

قام ديجول بجولة في المستعمرات الأفريقية في الفترة من اY - جب أغسطس 1901 لشرح أهداف دسنور الجمهورية الفرنسية الخامسة وإقناع الثعوب الأفريقية بالموافقة عليه (9")، وكان برفقة ديجول كل من بيير ب ـ مليملان Pierre P.flimlin رئيس وزراء Bernard Cornut- سابق ووزير دولة في حكومة ديجول ، برنارد كورنيه - جانتيل Gentille

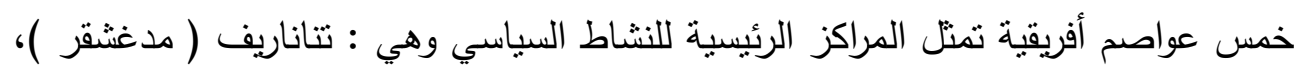
برازافيل ( الكونغو الفرنسية) ، أبيدجان ( ساحل العاج ) ، كوناكري ( غينيا الفرنسية ) ،

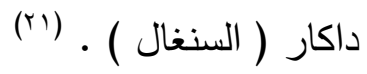
وكان يهذف ديجول من هذه الرحلة إلى إقناع الزعماء الأفارقة في هذه الأقاليم

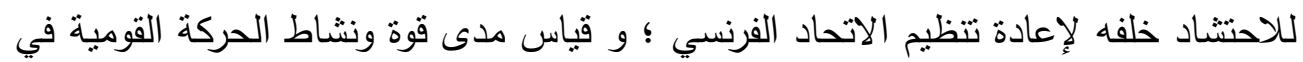

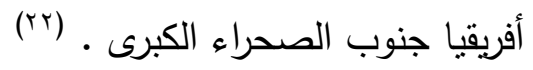


صرح ديجول أمام جمع حاثد من الصحفيين في باريس قبل الذهاب إلى أفريقيا في أغسطس 1901 : " أن الأقاليم الفرنسية فيما وراء البحار ليست دولا بالمعنى المفهوم وأنه من الواجب أن نوجد نوعا من الحكم الفيدرالي يجمع بينتا وبينهم ، ولو أنني أفضل أن أجعل علاقتنا المستقبلة تقوم على أساس كونفدرالي ولو على الأقل بالنسبة لبعض الأقاليم مثل ولث لهن

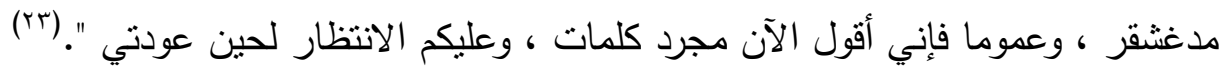

\section{موقف الثبب الفرنسي والأحزلب من دستور ديجول :}

بعد أن انتهى ديجول من جولته في المستعمرات الأفريقية ، قام بجولة أخرى بين

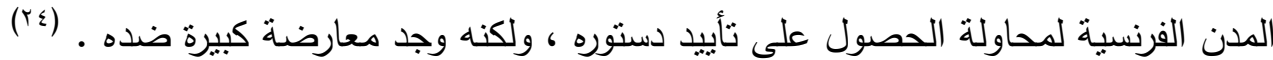
وبدأت الأحزاب السياسية الفرنسية بتتظيم سلسلة من الاجتماعات لتحديد موقفها

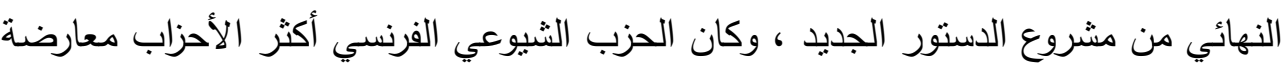
للاستور (ro)، كما قررت أيضا اللجنة التتفيذية للحزب الاشتراكي الذي كان يرأسه فرانسوا

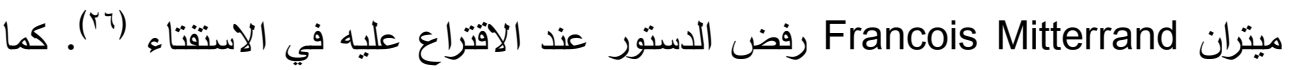
أعلن منديس فرانس Mendes France الزعيم الاتشتراكي الراديكالي في مؤتمر صحفي أنه

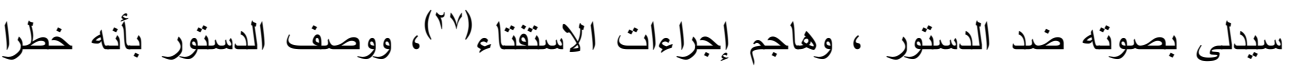

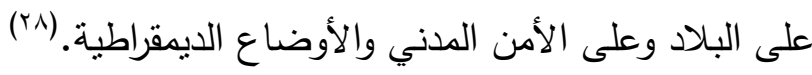

\section{ديجول في غينيا :}

لقد شعر ديجول بأنه نجح في فرض فكرته على الدول الأفريقية ولكنه صُدم عندما

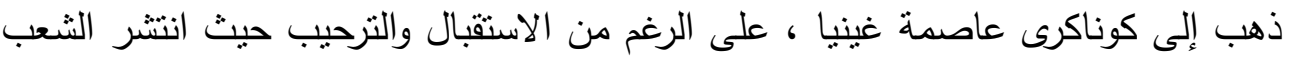
الغيني على طريق المطار وهم يرقصون رقصاتهم الوطنية ، ومر موكب ديجول وبجواره

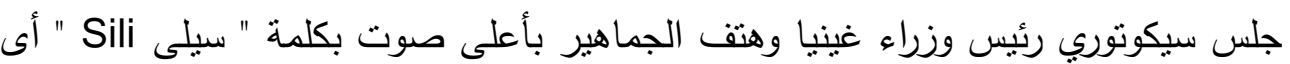

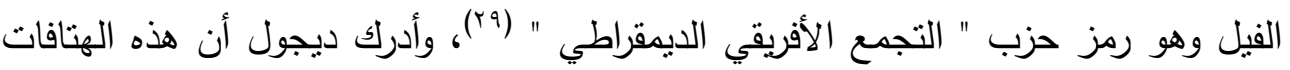
ليست له وإنما للزعيم الغيني سيكوتوري فقد كان ديجول على علم بالمشاعر المعادية للفرنسيين في غينيا وإصرارهم على طلب الاستقلال. (·r) 
وقد ذهب ديجول إلى كوناكرى في 0 أغسطس 1901 لإجراء مباحثات وللتحدث

مع الغينيين حول الدستور ، وكان معه الحاكم الفرنسي بيير ميسمير Pierre Messmer ، وكان على سيكوتوري رئيس وزراء غينيا أن يلقي خطابا تمهيديا يوضح فيه أرائه السياسية

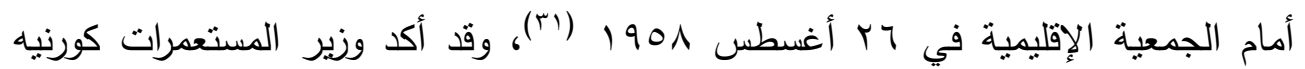
جانتيل لديجول أن سيكوتوري سيتقبل ما قبله غيره ولهذا فإنه يعثقد أن الثعب الغيني سيوافق على دستوره . (r) بدأ سيكوتورى خطابه بالهجوم على الإدارة الاستعمارية الفرنسية ثم أستمر بعد ذلك

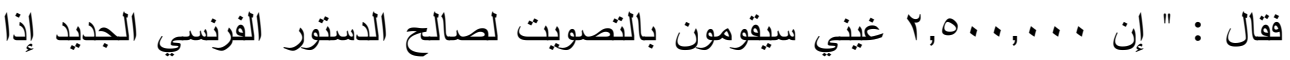

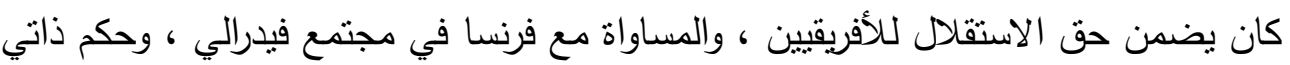
داخلي كامل وإتحاد الأقاليم الأفريقية في وحدة كبيرة واحدة " ، ورد عليه ديجول غاضباً :

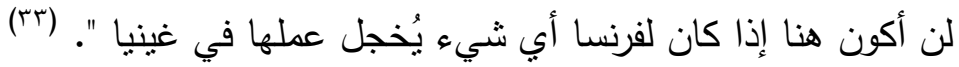
وذكر سيكوتوري في خطابه أيضا : " إن غينيا تطمح إلى أن تظل في المنظمة ست سنوات تقريبا ، على أن تكون عضوا في المنظمة بكل معنى الكلمة ، وذللك لأنها تطالب بالحق الكامل في تقرير المصير وهذا يعني القضاء على أثر الاستعمار في الإدارة

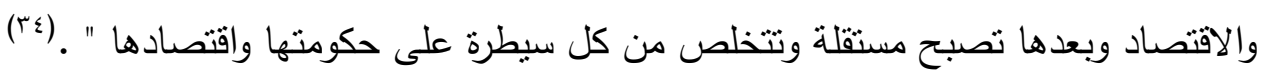
أندهش ديجول لهذه الثروط وغادر الاجتماع غاضبا وأعتذر عن حفل الغداء الذي

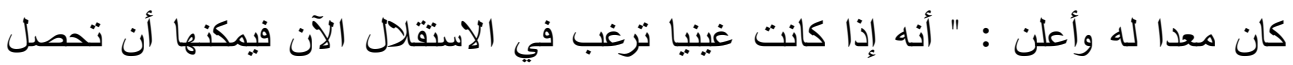

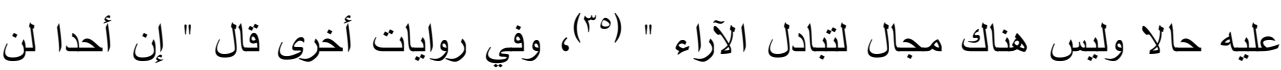

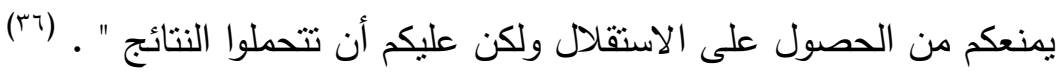
غضب سيكونوري وشن هجوما على ديجول ، وعبأ الثعب ضده وضد دستوره الجديد وأعلن : " أننا نفضل أن نكون أحرارا فقراء .. على أن نكون عبيدا أثرياء .. أننا مواطنون أفريقيون ونريد أن نبقى مرتبطين بفرنسا .. وللآن نُعامل معاملة تخلو من العدل

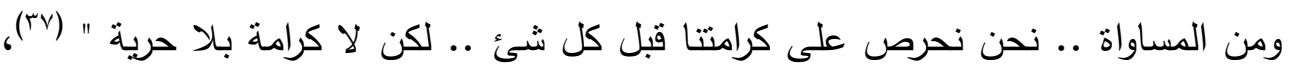
فرد ديجول قائلا " إن كل ما عليك أن تفعله هو أن تصوت في الاستفتاء بلا أو بنعم • وإني

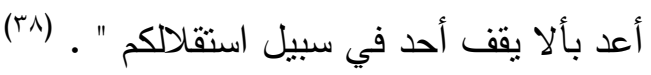


وفي Y أغسطس 1901 أب قبل الاستقاء بشهر نم إذاعة خطاب سيكوتوري وقال فيه: " علينا أن نذكر أن المجلس الأعلى في أفريقيا الغربية الفرنسية قد صوت ثنلاث مرات ضد الد جعل أفريقيا بلقانا أخر • وأن هذا الدستور الجديد لم يراع أنه يؤدى إلى تقسيم تعسفي ، ويعوق

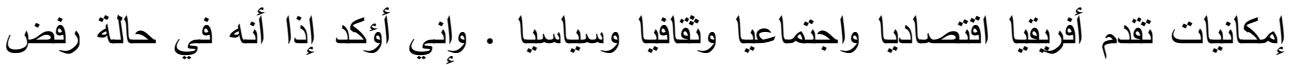
الحكومة الفرنسية لأمال أفريقيا المشروعة فإن مشروع فرنسا الدستوري هذا سوف يراعي

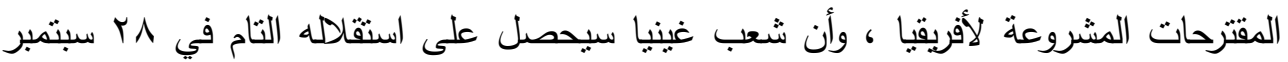
المقبل ، وسيطالب بمباحثات مع فرنسا على أساس عقد اتفاقية مشتركة بينهما ـ وفي حالة رفض فرنسا ستقرر غينيا دون أن تضيع وقتها طريقا أخر لمصيرها على أن يكون هذا عالطئ الطريق الجديد يتيح لها أكثر وأكثر بأن تستمر في كفاحها بنجاح في سبيل تحرر وحدة أفريقيا"(؟؟) . بعد ذلك بأسابيع في ع ا سبتمبر 1901 ، التقى قادة التجمع الديمقراطي الأفريقي في كوناكري للتوصل إلى سياسة مشتركة للتصويت في الاستفتاء ، ودعا هوفويت بواينى قادة الأحزاب الأفريقية إلى التصويت بنعم في الاستقتاء وبالفعل أيده في ذللك كل القادة وأعلنوا ثقتهم في ديجول ، وتعهدوا بالتصويت لصالح الدستور (·؛). أما عن سيكوتوري فقد رفض دعوة هوفويت ، وأعلن رفضه للاستور • وقد عبر عن ذللك في المؤتمر الوطني للحزب الديمقراطي الغيني في نفس اليوم وقال : " إن اختبارنا للاستقلال لم يكن يمثل إرادة غينيا وحدها ، وإنما كان هذا الاختيار على العكس يهدف إلى تعجيل الحصول على الاستقلال حتى لتلك الدول التي تميل إلى الانضمام إلى (المجموعة

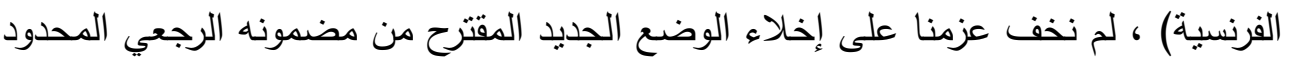
وتحويل هذا الجهاز المقصود منه القضاء على تعبئتنا السياسية إلى عنصر حركي لتحقيق استقلال البلاد الأفريقية الخاضعة للحكم الفرنسي • إن موقف غينيا الباسل قد قوض بالفعل

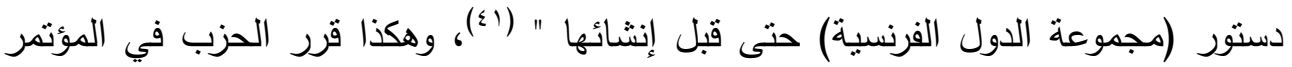

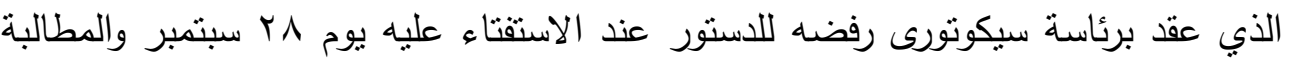
بالاستقلال التام بالإضافة إلى أنه أعلن أن الدستور يسعى لفرض السيطرة الفرنسية على

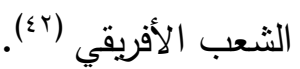


وهكذا بدأت معركة الاستفتاء على الدستور الجديد فقام الفرنسيون بالدعاية لدستورهم

وتحريض الشعب للموافقة عليه واستخدموا في ذلك كل الوسائل والإمكانيات التي لايهر (rاء).

وفي 0r سبتمبر 1901 قبل الاستفتاء بأيام قال سيكوتورى في إحدى خطبه القومية

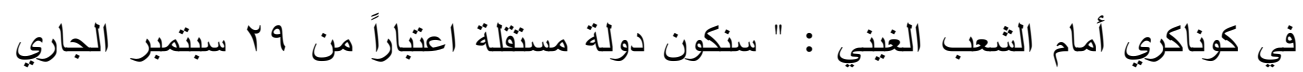
فسوف نصوت بالرفض في الاستفتاء بشأن المجموعة الفرنسية ، التي ليست في الواقع سوى الاتحاد الفرنسي القديم في صورة مجددة ، سوف نرفض عدم المساواة ، وعدم المسئولية ، وابتداءً من وج سبتمبر سنصبح دولة مستقلة ، ونتحمل المسئولية الكاملة الثاملة لجميع شئوننا. وسوف ننضم دولة جديدة إلى قائمة الدول الحرة ، وسيصبح يوم Y سبتمبر يوم

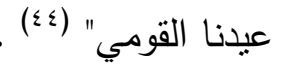
يوم الاستفتاء :

تحدد يوم Y سبتمبر 1901 للاستفتاء على الدستور الجديد للجمهورية الفرنسية

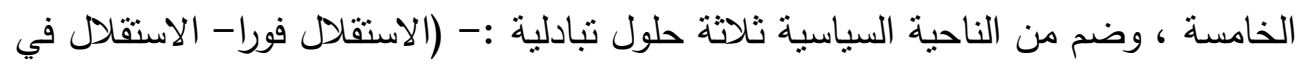
المستقبل طبقا لإجراءات حددها الدستور الجديد - عدم الاستقلال عن فرنسا) ، ومن الناحية الاقتصادية فقد خير الدستور الجديد المستعمرات الفرنسية بين احد أمرين: إما الاخول في هي

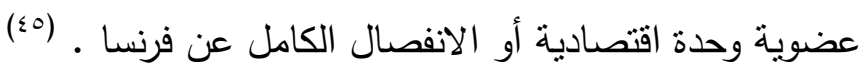

ونم الاستعداد للاستفتاء التاريخي في الأقاليم الأفريقية ، ففي غينيا بلغت مراكز

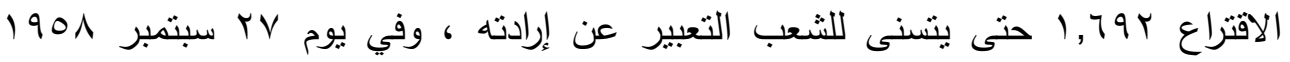

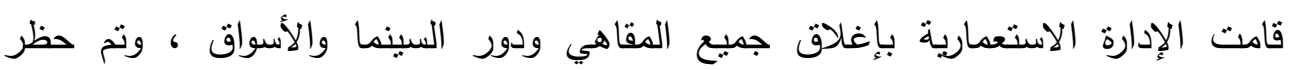

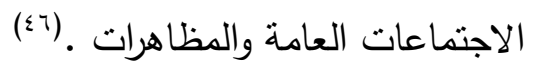

وفي صباح يوم الاستفتاء شهدت الساعات الأولى إقبالا كبيرا بشكل منظم في جميع

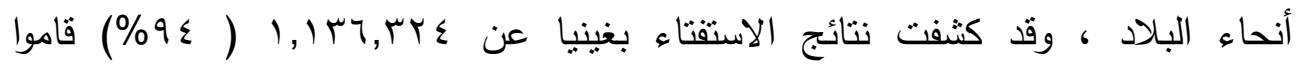

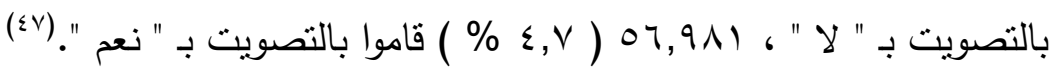

أعلن سيكوتورى رئيس وزراء غينيا في أول مؤتمر صحفي يعقده بعد الاستفتاء : " أن بلاده سترسل وفدا لإجراء مفاوضات في فرنسا إذا نأكدت أن فرنسا سنستقبل هذا الوفد . 
ثم قال أن فرنسا ليس لها من الناحية الأدبية أي حق في استغلال العجز الاقتصادي لبلد

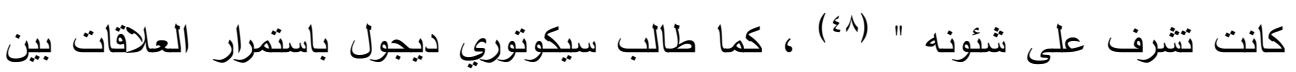
البلدين بموجب المادة N1 من الدستور الجديد والتي تتص على : " الدولة التي تختار

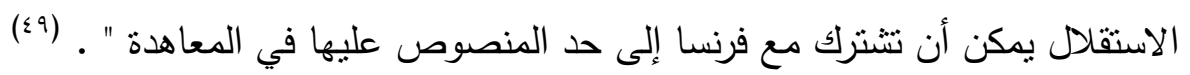
وعلى الرغم من تأكيدات سيكوتورى والتي جاء فيها : " إننا نريد الاستقلال بالتعاون مع فرنسا وليس بمعاداتها " إلا أن فرنسا قامت في نفس اليوم وهو إعلان الاستقلال باتخاذ

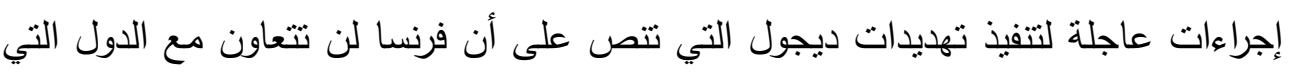
تخرج من الكومنولث. (0. (ن)

وعلى إثر ذللك قام رستروس Risterucci المبعوث الخاص لديجول بإبلاغ سيكوتورى بأن فرنسا ستسحب موظفيها البالغ عددهم ثلاثة ألاف موظف خلال أيام ، وأنها ستوقف منحتها السنوية لغينيا وقدرها عشرة ملايين جنيه إسترليني ، ثم أوققت فرنسا أعمال الإنشاء الخاصة بالطرق والمدارس ، و أوققت معاملاتها التجارية مع غينيا فقد كانت فرنسا

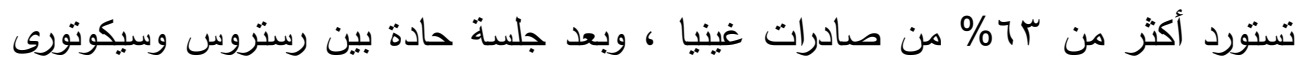
أعلن المبعوث الفرنسي أن فرنسا ستقطع جميع اتصالاتها مع حكومة غينيا. (1) فقد حاول المستعمرون الفرنسيون معاقبة الثنعب الغيني لمطالبته بالاستقلال ، ولذلك عمدوا إلى تخريب الاقتصاد وأوجه النشاط المختلفة في غينيا ، ومن مظاهر ذللك أن أستدعى دانى

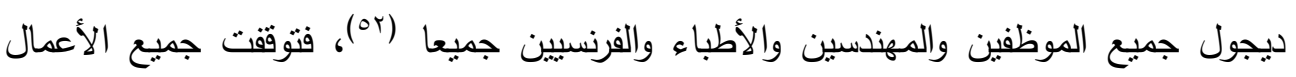
وخلت البنوك ، كما قام بعض الفرنسيين بقطع التوصيلات الكهربية والتلافونية وإتلاف جميع

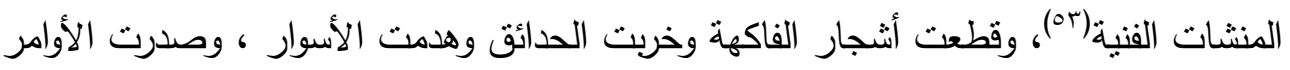

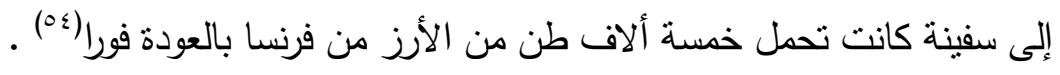

وهكذا وجدت غينيا نفسها على حافة كارثة حيث استمر الفراغ السياسي والاقتصادي

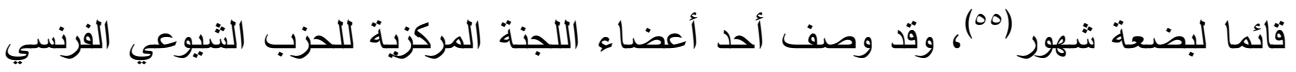
هذه الإجراءات بأنها :" لا تعدو أن تكون بلطجة سياسية وضغطا على شعب غينه ولينيا

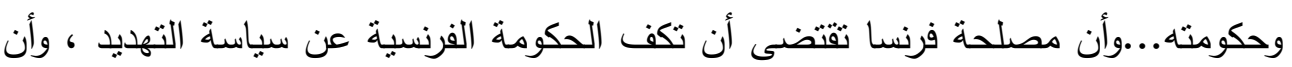

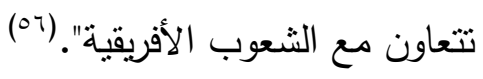




\section{اعلان استقلال غينيا :}

في اليوم الثاني من شهر أكتوبر عام 1901 اجتمعت الجمعية الإقليمية لغينيا لأول

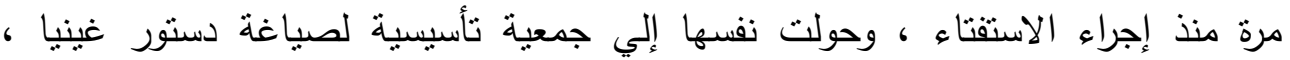
وأعلنت الجمعية استقال غينيا وأصبحت جمهورية ديمقراطية شعبية مستقلة ، وعلى إثر ذلك التكاه

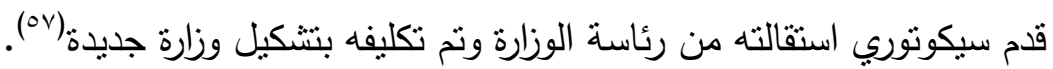

كما صرح سيكوتورى في نفس اليوم باعتباره سكرتيرا للحزب الديمقراطي الغيني تصريحا جاء فيه : " إن شعب غينيا الذي عبر عن رأيه بكل صراحة مازال مستعدا لتحمل فئل

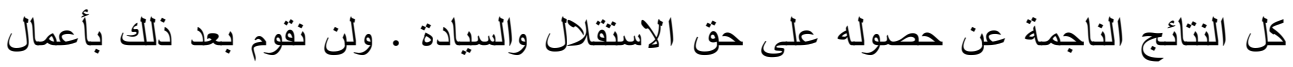
غير مستساغة حيال فرنسا. على أن يكون هذا في نطاق كيان الدولة واستقلالها

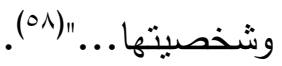

وبلغ عدد الدول التي اعترفت بغينيا أكثر من ثلاثثن دولة منها الجمهورية العربية المتحدة وحكومة الجزائر والاتحاد السوفيتي ويوغوسلافيا بجمهورية غينيا والسويد وبريطانيا

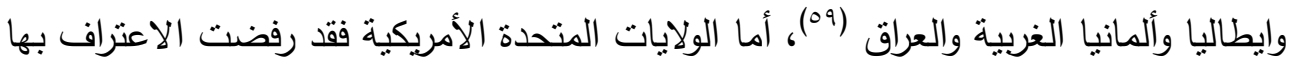

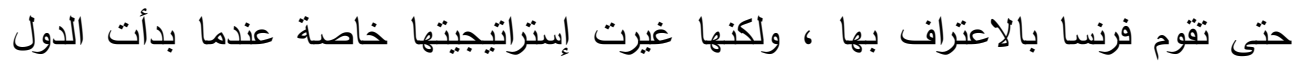

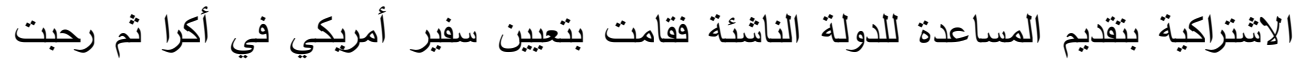

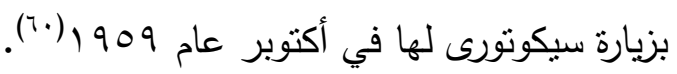
وفي أواخر نوفمبر قام سيكوتورى بزيارة غانا ، وكون معها اتحاد مشترك (آن)، وفي

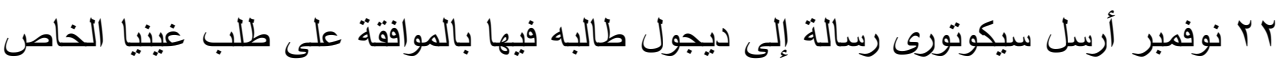

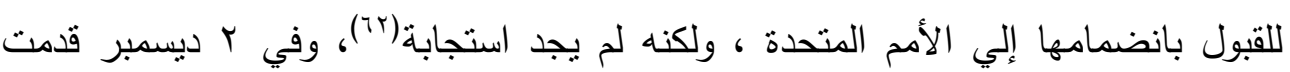

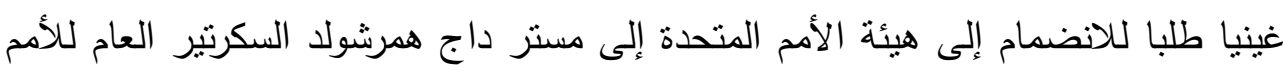

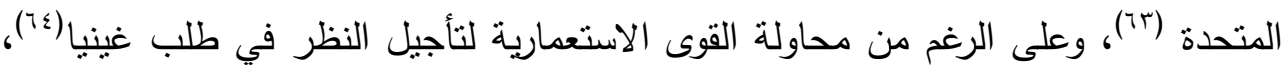

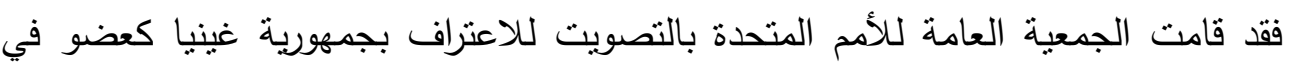
rا ديسمبر ، وقد وافق كل المندوبين على قبولها عضوا بإستشاء فرنسا التي امتتعت عن التصويت(70). وهكذا أصبحت غينيا العضو الثاني والثمانين في هيئة الأمم المتحدة (II'). 


\section{الخاتمة}

إن استقلال غينيا يعتبر في الواقع أول تصدع خطير تصاب به جدران الإمبراطورية الفرنسية في أفريقيا ، وبداية الانتصار لحركة التحرر الوطني بالقارة الأفريقية . وقد تبين من خلال هذه الدراسة أنه نظرا للتيارات التحررية التي ظهرت واشتدت بعد الحرب العالمية الثانية ، لجأت فرنسا إلي تغيير الدستور الفرنسي وإيجاد وضع جديد للاتحاد الفرنسي ، وقد كانت فرنسا واتقة من موافقة الأقاليم الأفريقية على مشروعها الدستوري ،

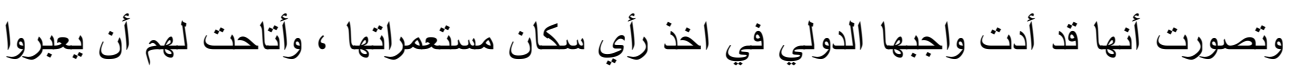
بحرية عن رغبتهم في الانتماء إليها .

ولكن الثعب الغيني فهم هذه المؤامرة التي دبرتها فرنسا ، وتحدى ديجول برفض

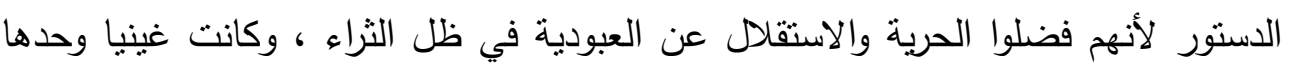

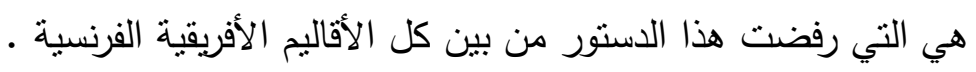

وتعرضت غينيا إلي فترة حرجة بعد الاستقلال نتيجة لما قام به ديجول تجاه غينيا ، فقد انقطعت العلاقات بين غينيا وفرنسا ، ونم إلغاء المعونة التي كانت غينيا تتلقاها من فرنسا ، وسحب الموظفين الفرنسيين منها ، ولكن على الرغم من ذلك تحدى سيكوتوري هذه

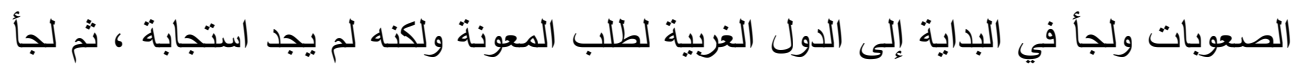
بعد ذلك إلى الكتلة الثرقية مما أدي إلى اتهامه بالثيوعية ، ولكنه أجاب على هذه

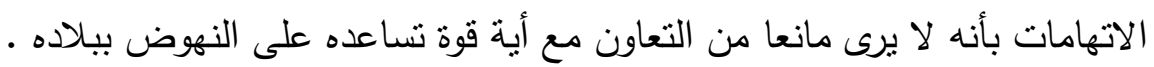

وأكد سيكوتورى على ضرورة الوحدة الأفريقية قبل الاستقلال للتوصل إلى تحرر

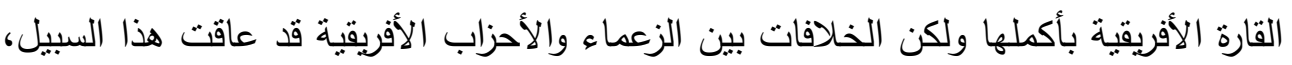

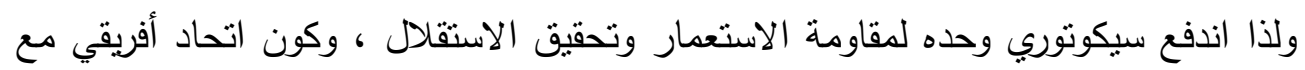
غانا برئاسة كوامي نكروما في نوفمبر عام 901 الكي يكون نواة لوحدة الدول الأفريقية . 


\section{الهوامش}

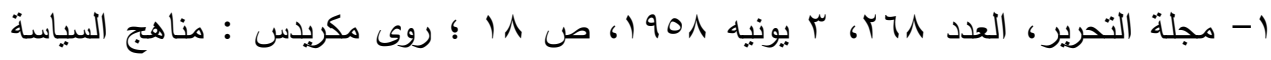
الخارجية في دول العالم، ت : حسن صعب، طץ، مؤسسة فرنكلين للطباعة والنشر، دار

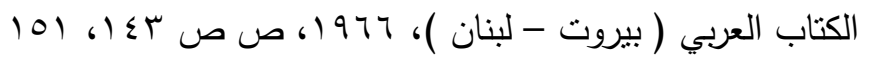

Y - وزارة الخارجية، سفارة جمهورية مصر بمدينة بروكسل، بشأن : نطور المسألة الفرنسية، ؟

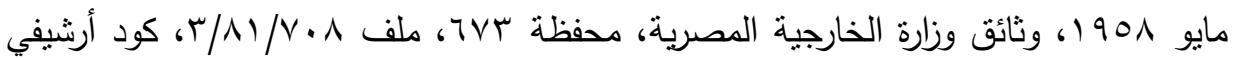

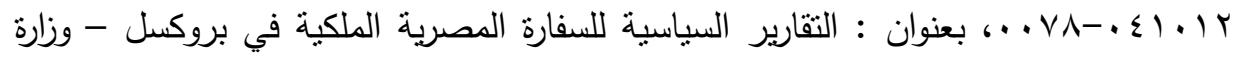

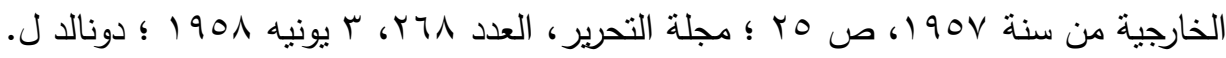
وايدنر : تاريخ أفريقيا جنوب الصحراء، ت : شوقي عطا اله الجمل، ج ب، مؤسسة سجل

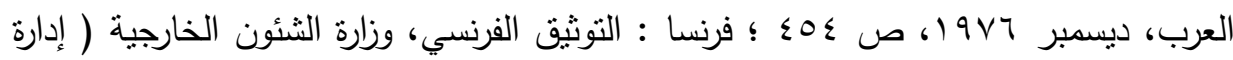

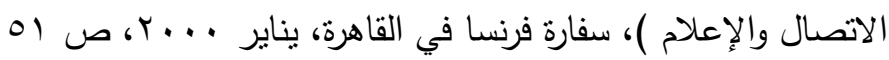
r - الرأى، السنة الرابعة، العدد بلا، ب يونيه 1901، ص ص (، 9 ع - وزارة الخارجية، الإدارة السياسية، إدارة غرب أوروبا، بشأن : بحث عن فرنسا، مب سبتمبر

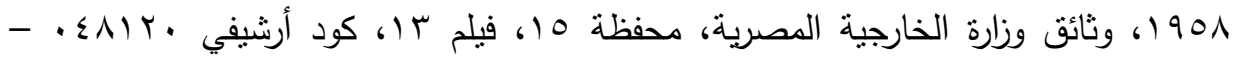

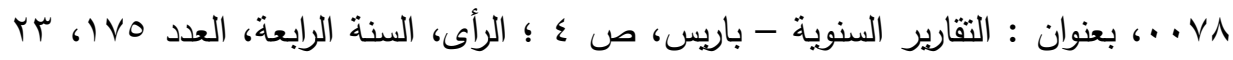
يونيو 1901، ص صعون

0 - موريس دوفرجيه : دساتير فرنسا، ت : أحمد حسيب عباس، مراجعة : السيد صبري،

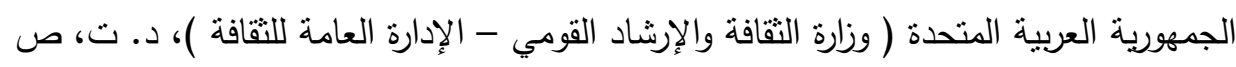

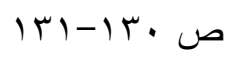

4 - شوفي الجمل : تاريخ كثف أفريقيا واستعمارها، مكتبة الأنجلو المصرية، أبريل اY919، ص ory

V - شوقي الجمل، عبد اله عبد الرازق إبراهيم : تاريخ أفريقيا الحديث والمعاصر، دار الثقافة -

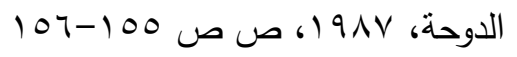

^ - صلاح العقاد : الجمهوريات الأفريقية في الاتحاد الفرنسي، الدار القومية للطباعة والنشر،

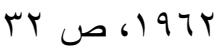


9 - حمدنا اله مصطفى حسن : أحمد سيكوتورى ( بين القومية الأفريقية و الانتماء الإسلامي)،

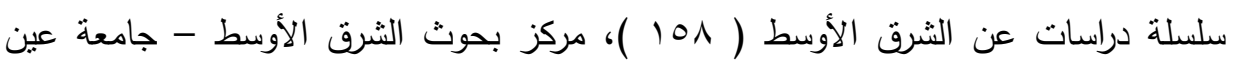

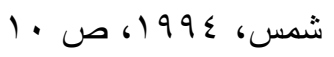

• 1 - وزارة الخارجية، الإدارة السياسية، إدارة غرب أوروبا، بثأن : بحث عن فرنسا، ص ؟

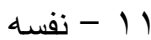

Y I - عبد الملك عودة : سنوات الحسم في أفريقيا .197 - 1979، مكتبة الأنجلو المصرية،

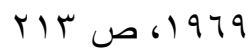

r ا سفارة الجمهورية العربية المتحدة - أكرا، بشأن : موقف المستعمرات الفرنسية في غرب

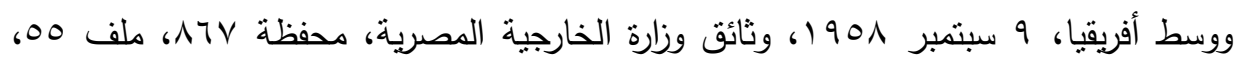

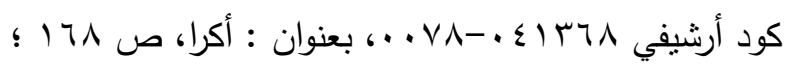

Sik, Endre : The History Of Black Africa ,Translated by : Sandor Simon, Vol. IV , Akadémiai Kiado, Budapest, I9V ع ا - سفارة الجمهورية العربية المتحدة - أكرا، بشأن : انعقاد مؤتمر الأحزاب الأفريقية بمدينة

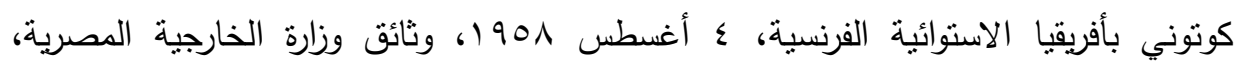

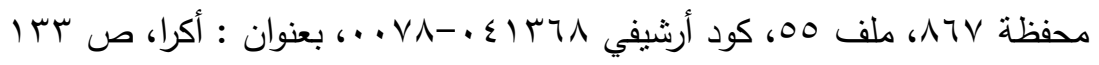

10 - Barraclough , G.: Survey of International Affairs 1907 - 1901, Oxford University Press, New York, 197 r, P. $\{01$

17 - وزارة الخارجية، الإدارة السياسية، إدارة غرب أوروبا، بشأن : بحث عن فرنسا، ص ص ب -

IV - فرانتز فانون : من أجل أفريقيا، ت : محمد الميلي، طا، منشورات المطبوعات الوطنية

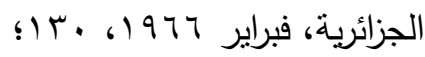
The PRA, " New Force in FWA " , (Africa Today, Vol. ๑ , No. ๑, September - October 1901$)$, PP. I r-IT 11 1 - وزارة الخارجية، الإدارة السياسية، إدارة غرب أوروبا، بثأن بحث عن فرنسا، ص ع 
19 - Grimal, Henri : Decolonization ( the British, French, Dutch and Belgian Empires 1919-1974), Translated by : Stephan De Vos, Routledge and Kegan Paul, London and Hanely, 1970, P.rV

r. - Du Bois , Victor D. : The Guinean Vote for Independence " The Maneuvering before The Referendum of September $r \wedge, 190 \wedge$ ", AUFS Reports, West Africa Series, Vol.V, No.V , New York : AUFS, $197 \mathrm{r}$ , P. 1

Y) - Delavignette, Robert : L' Afrique Noire Francaise et son destin , Gallimard, 19ะ7, PP.1r^-1 rq; The New York Times, August T., 1901, P.1.

rr - Du Bois : The Guinean Vote for Independence, P. I

$$
\text { ب r - فوزي عبد الحميد : المرجع نفسه، ص ع }
$$

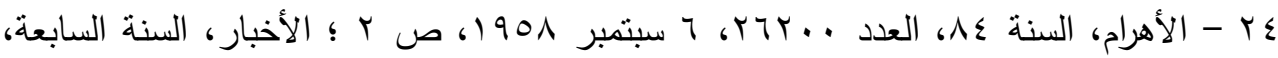

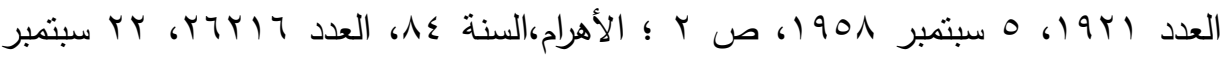

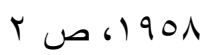

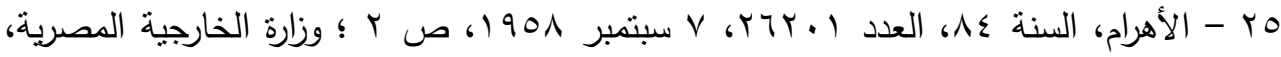

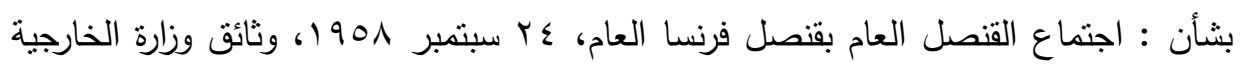

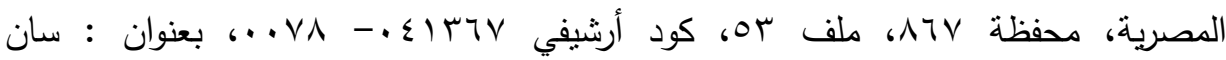
فرانسيسكو عام 1901 1، ص صنه

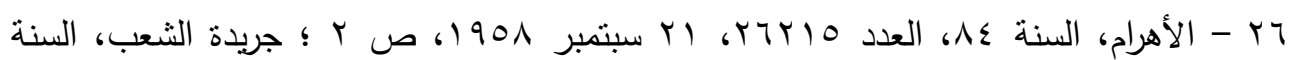

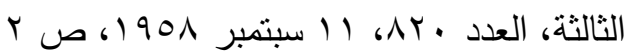

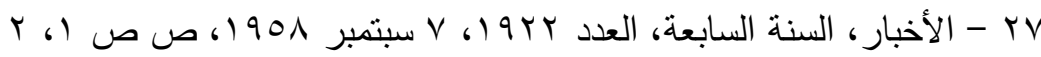

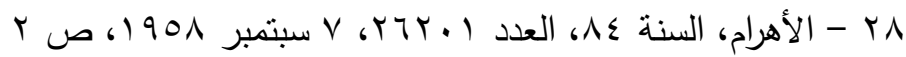


rq - Schmidt , Elizabeth : " Top Down or Bottom Up? Nationalist Mobilization Reconsidered, with Special Reference to Guinea (French West Africa) " , ( The American Historical Review , Vol. 11. , No.乏, October $Y . .0)$, P. $9 \vee 4$

r. - Barraclough, G. : Op.Cit, P. $\_$

اس - رولف إيناليندر : عشرة رجال من أفريقيا ( حياتهم - أعمالهم - أهدافهم )، ت : أحمد

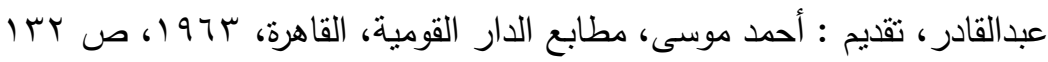

r - جويندولن• م كارتز : الاستقلال لأفريقيا، ت : دانيال عبد اله رزق، مراجعة محمود شوقي

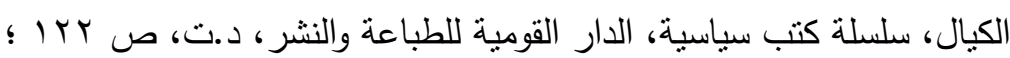

Corfield, Justin : Guinea (Africa and the Americans " Culture , Politics, and History", Edited by : Richard M.Juang and other, Vol. I,

California, , r.. , P. or $\varepsilon$

r - The New York Times, August rq,1901 , P.A ; Carter, Gwendolen M. : African one-party States, Ithaca, New York, 197 r, P. I TV ; Du Bois, Victor D. : Guinea, in : Poitical Parties and national Integration in Tropical Africa, Edited by : James S. Coleman and other , University of California Press, London $197 \varepsilon$, P. $19 \varepsilon$

ع - - رولف إيتاليندر : المرجع نفسه، ص بrا ؛ زاهر رياض : استعمار القارة الأفريقية

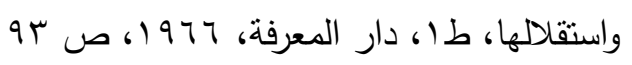

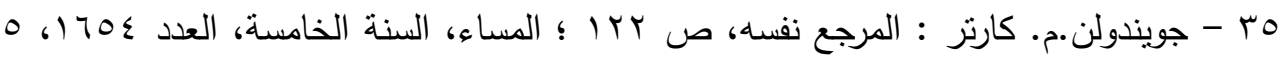
مايو (971، ص ب ؛ شوقي عطا الله الجمل ؛ عبد الله عبد الرازق إبراهيم : رواد التحرر

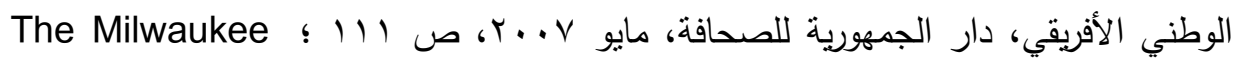
Journal, December $v, 1901$, PP. $1-r$

rч - Carter , Gwendolen, M.: Independence for Africa, Thumaes and Hadson, London , 1971, P. IrT; 


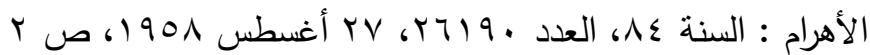

rv-Lusignan, Guy De : French-Speaking Africa Since Independence, Pall Mall Press, London , 1979 , P. Yr ; Goss , Hilton P. : The Political future of the Independent Nations of Africa , Tempo , General Elictric Company, California , I August , 197., P. I

^ץ - ناصر • تورى. سوكارنو ، إعداد : لجنة كتب سياسية، الكتاب Y Y Y، مطابع الدار القومية،

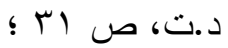

The Leader-Post, No.r.. , August r $7,190 \wedge$, P. 1

q - كلود فوتييه : المرجع نفسه، ص ^ץ إ؛

The Evening Independent, Vol. r, No. rrq, October 1,1901, P.r

乏. - Du Bois : The Guinean Vote for Independence , P. ๑ ; Lusignan : Op. Cit , P. Y $\leqslant$

اء - أحمد سيكوتورى : تجربة الثورة في غينيا، ت : نور الدين الزرارى، إثراف وتقديم : عبد

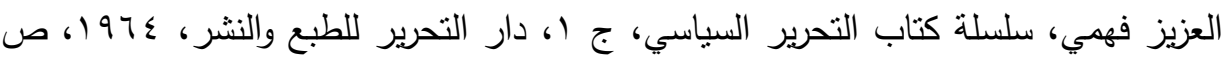
511

Barraclough : Op.Cit, PP.£o1- sor; The New York Times, September 10,1901, P.Y

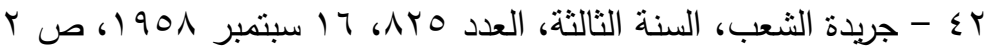
بـ - سفارة الجمهورية العربية المتحدة - أكرا، بشأن : موقف المستعمرات الفرنسية في غرب ووسط أفريقيا، 9 سبتمبر 1901 1، ص ع

ء - صلاح صبري : أفريقيا وراء الصحراء، سلسلة الألف كتاب ب. ب، إدارة الثقافة العامة بوزارة

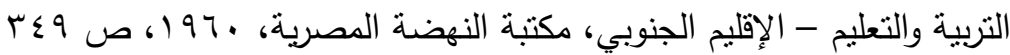


0؛ - سفارة الجمهورية العربية المتحدة - أكرا، بشأن : موقف المستعمرات الفرنسية في غرب

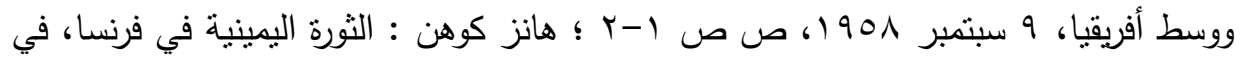

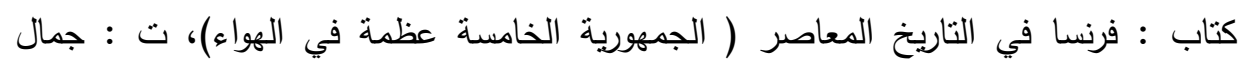

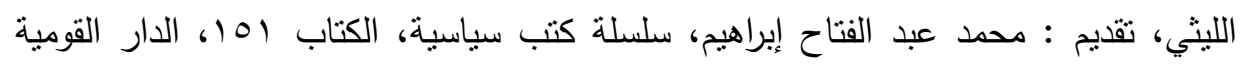

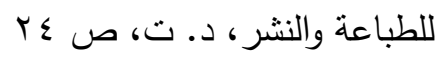

$\Sigma 1-$ Du Bois : The Guinean Vote for Independence, PP. $1-\vee$

$\sum \vee-$ Schmidt, Elizabeth : "Cold War in Guinea, The Rassemblement Démocratique Africain and The Struggle over Communism ( 190.$190 \wedge$ ) " , ( Journal of African History, Vol. $\leqslant \wedge$, No. I, March r... ) , P.119

§^-Lewin, André : ler Octobre 190^. L'incompréhension de Paris, in :

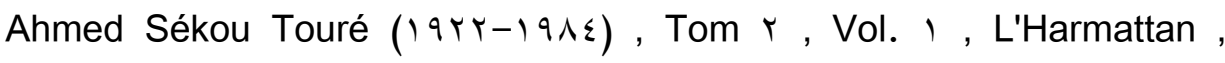
Paris , Y. . , (www.webguinee.net) ; Lewis , William H. : FrenchSpeaking Africa ( The Search for Identity ), Walker and Company , New York, 1970, P. ro; The New York Times, October 1, 1901 , P. 17

§q- The Evening Independent, No. rrq, October 1, 1901 , P. ; Carter : African one-Party States, P.IVI; The New York Times, October $r$ , 1901, P.0

•0 - أحمد صوار : سيكوتوري، سلسة كتب سياسية، الدار القومية للطباعة والنشر، اب مايو

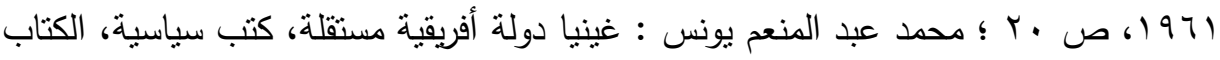

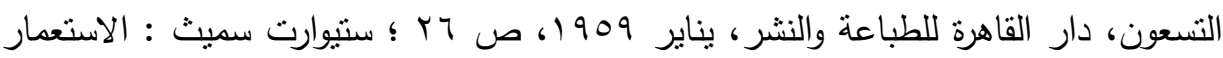

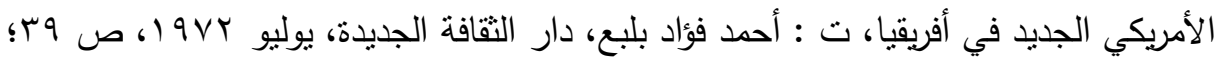

The New York Times, October r, 1901, P.o 
اه - شوقي عطا اله الجمل ؛ عبد اله عبد الرازق إبراهيم : رواد التحرر الوطني الأفريقي،

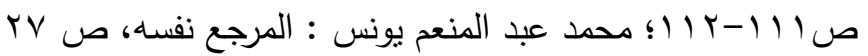

or - Derman , William : Cooperatives in the Republic of Guinea Problems of Revolutionary Transformation ", in : Popular Participation in Social Change, Edited by : June Nash, Mouton and Co Publishers, Paris, $19 \vee 7$, P. $\leqslant 1 \leqslant$

or - Singer, Barnett ; Langdon, John W. : Cultured Force (Makers and Defenders of the French colonial Empire ), The University of Wisconsin press, $, r . . \varepsilon, P . r \leqslant \wedge$

عه - رولف إيتاليندر : المرجع نفسه، ص بrا ؛ زاهر رياض : استعمار القارة الأفريقية

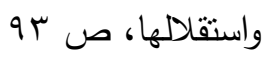

0ه - رولف إيتاليندر : المرجع نفسه، ص سبا

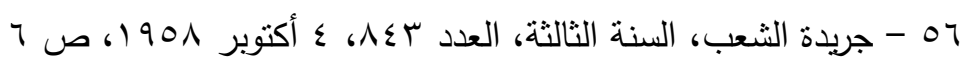
ov - "Proclamation de L'independence de la République de Guinée " , (Journal official de la République de Guinée, Débats Parlementaires , 1 Année, No. 1, Octobre 1901), PP.1-r ; Du Bois : The Guinean Vote for Independence, P.9; The Edmonton Journal, No. rVr, October r, 1901, P.0;

$$
\begin{aligned}
& \text { الأهرام، السنة عی، العدد VYT }
\end{aligned}
$$

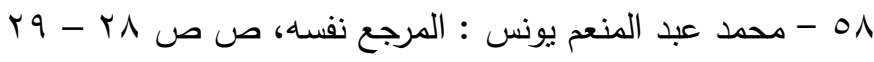

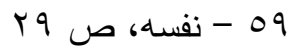

7. - Menorandum From Secretary of State Dulles to President Eisenhower , October M1,1901, in : FRUS 1901-197. , Vol. XIV (Africa), Washington, 1994, PP. $7 \vee 9-4 \Lambda$. ; Letter from the President of the 
United States (Eisenhower) to the Prime Minister of Guinea (Touré), November 1,1901, in : American Foreign Policy, Current Documents 1901, Historical Office, Bureau of Public Affairs, April 197r, P.1.90

$7)$ - Schmidt : Cold War and Decolonization in Guinea 19 $17-1901$, P.IVฯ; Lusignan : Op.Cit, P.r

7r - Schmidt : Cold War and Decolonization in Guinea 19 $17-1901$, P.IV9

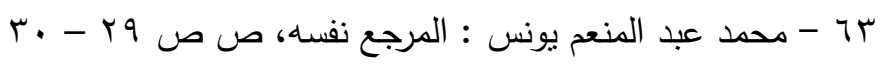

$7 \varepsilon-$ Schmidt : Cold War and Decolonization in Guinea 19 $17-1901$, P.।v1; The New York Times, 1. December 1901, P. 10

10- United Nations, Security Council, 9 December 1901, Admission Of New Members, S/₹Irr ; Admission of The Republic of Guinea to Membership in The United Nations, Resolution Irro (XIII), December $1 Y, 1901$, in : American Foreign Policy, Current Documents 1901, Historical Office, Bureau of Public Affairs, April 197r, PP. I Tr, I I

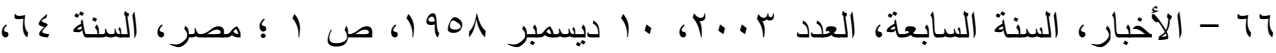

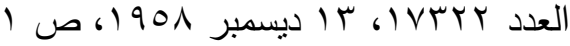

